\title{
Proyectos educativos regionales y proyectos de nación divergentes
}

\author{
Luis Arturo Ávila Meléndez \\ CIIDIR Michoacán IPN, México
}

\begin{abstract}
Resumo
Proponemos valorar la participación de actores regionales en la construcción de ciudadanías nacionales con matices regionales mediante la creación de instituciones de educación superior de régimen privado. Los proyectos de nación de los actores regionales cuentan con una base social regional cuya historicidad se ha ido construyendo a través de procesos conflictivos entre grupos regionales de clase y en interacción con agentes del gobierno nacional. Pretendemos mostrar que los principios promovidos al interior de las instituciones sobre la realidad social y política, el significado del compromiso político y la justicia, están ligados a los contrastantes resultados en cada región de procesos conflictivos de larga duración, como la concreción de pactos entre grupos regionales culturales y de clase durante la consolidación del Estado post-revolucionario.
\end{abstract}

Palabras clave

Educación superior privada; Cultura regional; Estado-nación

\section{Proyectos educativos y Estado nacional en México}

El análisis de la conformación de los Estados nacionales modernos de Latinoamérica ha identificado la tentativa de los gobiernos nacionales de conformar, a través del sistema educativo público (Galván, 2004; Rockwell, 2007), un proyecto de unificación y homogenización ideológica en torno a la unidad nacional, que supuestamente favorecería la igualdad de 
oportunidades, supuesto que palidece ante la perturbadora desigualdad real prevaleciente en la mayoría de los países de la región. Se han señalado las limitaciones de los mecanismos desplegados en las instituciones escolares, que en muchas ocasiones sólo ha promovido un sentimiento patriótico y dejado en segundo plano la conformación de ciudadanos informados y comprometidos políticamente con una sociedad nacional pluricultural (González, 2008) ${ }^{1}$.

Igualmente, se ha identificado que la formación de una identidad ciudadana nacional ha ocurrido principalmente a través de la participación de los pobladores en procesos de organización política fuera de la escuela (Correia, Castro y Gouvêa, 2007). Por ejemplo, los estudios sobre lectoescritura en zonas rurales ha identificado los usos de documentos 'oficiales' en conflictos por la tierra desde el siglo XVI, lo que señala el uso de la lectoescritura en contextos no escolarizados sino en el ámbito de la esfera pública que propiciaron la construcción de una ciudadanía nacional activa (Rockwell, 2001; Jiménez y Smith, 2008).

Frente a la definición restringida de "ciudadanía" promovida por la escuela pública, hay que

reconhecer e valorizar o papel activo das pessoas e dos grupos na conformação dos Estados-nação ao longo do tempo (cf. Hattersley, 2006). Há, por isso, outras perspectivas e processos sociais de construção da cidadania. A cidadania é também (e sobretudo) uma conquista das classes populares e não uma mera concessão do Estado. (Afonso y Lucio-Villegas, 2007: 81).

En este sentido, proponemos poner atención al funcionamiento de instituciones de educación superior de régimen privado, para valorar la participación de actores regionales en la construcción de ciudadanías nacionales con matices regionales, derivados de una base social regional cuya historicidad se ha ido construyendo a través de procesos conflictivos entre grupos regionales de clase y en interacción con agentes del gobierno nacional (Holland y Lave, 1996). Espero demostrar que determinados procesos conflictivos nacionales de principios del siglo $\mathrm{XX}$ generaron las condiciones ideológicas, económicas y políticas que permiten cierto consenso y la generación de cierta coherencia simbólica que se concreta en las instituciones educativas contemporáneas, de manera contrastante en dos regiones del estado de Michoacán, México. 
Aún reconociendo las limitaciones de los mecanismos para generar algún tipo de compromiso cívico o formación ciudadana durante los estudios universitarios (Egerton, 2002), esta perspectiva sitúa este esfuerzo por generar coherencia cultural en las instituciones educativas de nivel superior dentro de procesos históricos regionales que, a nuestro parecer, complementan los debates generales en torno a las relaciones entre ciudadanía y educación escolarizada. En particular, complementa el debate en torno a las transfiguraciones de la ciudadanía nacional debida a diversos procesos de globalización (Davies, Evans y Reid, 2005), pues nosotros hacemos énfasis en la historicidad de las relaciones sociales regionales que participan de esta misma reconfiguración.

El aporte al debate de las relaciones entre instituciones educativas y proyectos políticos de nación, está en el tipo de análisis cultural que empleamos para entender el desarrollo de dos instituciones educativas de manera comparativa. Aunque el enfoque de la antropología cultural que retomo comenzó a desarrollarse desde principios de los años ochenta (Roseberry, 1982, Wolf, 1982), al menos en México esta corriente antropológica no es la predominante en la investigación educativa. En esta presentación pretendo mostrar que este enfoque es útil actualmente para analizar cuestiones en torno al rol de la educación escolarizada en la formación ciudadana, en un contexto de reconfiguración del Estado-nación como unidad política ante procesos de globalización del capital financiero y mercados laborales y el resurgimiento de identidades regionales, puesto que una de sus tareas es evidenciar las relaciones complejas entre significado, economía política y poder. Contrariamente a lo que realizan la mayoría de los estudios que pretenden dar importancia a la "cultura" como un aspecto importante para la comprensión de las instituciones y prácticas educativas, el acento no está puesto en "patrones culturales" peculiares de grupos culturales específicos, a la manera en que lo realizan quienes retoman a Geertz (1973). El acento se ubica en las relaciones sociales e interacciones históricas que se desarrollan entre grupos culturales diferenciados y en mutua influencia. Se considera fundamental la construcción dinámica de ideologías que articulan y median las desigualdades entre dichos grupos, ideologías constituidas a través de esas relaciones sociales que implican aspectos económicos, políticos y de significado (ideologías y representaciones). 
Esta consideración de aspectos económicos y políticos ligados al surgimiento e interacción de grupos culturales es lo que permite aproximarse a la constitución de campos sociales o culturas regionales a través del tiempo. La historicidad de las instituciones sociales en general, y las educativas en particular, puede comprenderse conociendo la posición que ocupan distintos grupos culturales en un campo social, y la relación que tiene dicha posición con las acciones intencionales de los actores regionales y sus consecuencias en las posibilidades de existencia y transformación de una institución. Como una primera aproximación a los procesos regionales pertinentes, en la primera parte del trabajo se muestra, con base en información estadística nacional y estatal, que amplios sectores de la población mexicana están siendo excluidos de una educación superior pertinente y útil para su desarrollo. Dicho panorama subraya las desigualdades existentes en un proceso nacional de expansión de la educación superior, y sirve como un marco general para aproximarse al análisis de dos regiones del estado de Michoacán, México. En la segunda parte del artículo se especifican brevemente las relaciones desiguales que tienen lugar en la actualidad entre distintos grupos culturales al interior de las dos regiones, relaciones constituidas a través de procesos políticos y económicos previos a escala nacional y local, y las consecuencias de dicha situación para la constitución de dos instituciones de educación superior (IES) privadas fundadas por actores locales. En esta segunda parte espero dejar claro que las ideologías regionales que participan en la constitución y desarrollo de dos IES no son simples "patrones culturales" que pueden ser transformados simplemente mediante la difusión de un discurso políticamente correcto en torno al compromiso cívico y la equidad. Las ideologías expresadas y concretadas en prácticas, formas organizativas y discursos, son una arista de las relaciones sociales regionales que tienen fundamentos económicos y políticos, que respaldan y dan vida a dichas ideologías.

En consecuencia, finalizo con una breve una reflexión sobre la participación de actores regionales orientados por ideologías que en ocasiones se contraponen, sin hacerlo claramente manifiesto, a la participación de actores con ideas divergentes a ellos en la constitución de un nuevo Estado mexicano. La perspectiva de análisis cultural que propongo, aplicado a una institución educativa, impide estudiar de manera separada los 
aspectos simbólicos o ideológicos y los aspectos económico-políticos de los procesos sociales vinculados con la emergencia y desarrollo de dicha institución. Por lo tanto, más que un análisis de ideologías o de patrones culturales, este estudio pretende ejemplificar una forma de analizar las políticas ontológicas de actores regionales contemporáneos en torno a un proceso complejo de reconfiguración del Estado y la ciudadanía en México.

\section{Acesso a la educación superior en México}

En México, alrededor de $22.7 \%$ de la población en edad de cursar estudios de licenciatura, entre 19 y 23 años, se encontraba en dicha situación (ANUIES, 2007). Dicho porcentaje es bajo en contraste con países económicamente poderosos, aún cuando en los últimos 20 años la matrícula total de licenciatura se ha incrementado aceleradamente, triplicándose entre 1980 y 2007 al pasar de 731291 a 2,150,146 estudiantes (Kent y Ramírez, 1998; ANUIES, 2001 y 2007).

Bracho (2002) señalaba que un pequeño sector de la población, el que contaba con mayores ingresos, era el que había accedido en mayor proporción a la educación superior en el lapso de 1984 a 1996. El porcentaje de jefes de familia del quintil superior, de mayores ingresos, con escolaridad media superior y superior aumentó de 32 a 55\%. En cambio, en el quintil inferior pasó de 0.3 a 2.2\%. Más recientemente, Torche (2007: 13 y 29) identifica que en México y Chile las oportunidades de acceso "no estaban igualitariamente distribuidas, favoreciendo principalmente a las personas con origen educacional alto que antes no accedían al nivel universitario".

La distribución geográfica de las instituciones también se relaciona con el acceso. En 2000, Michoacán era uno de los diez estados con mayor concentración de la matrícula de educación superior en su capital, Morelia (78.2\%). La universidad pública estatal, concentraba más de $85 \%$ de su matrícula en Morelia (Ríos 2001)².

Por otra parte, el sistema de educación superior mexicano se encuentra claramente segmentado, en el sentido de que las características del origen social, situación económica y condiciones de estudio en casa de la mayoría de los estudiantes de las universidades públicas contrasta con las correspondientes de las universidades privadas. En las privadas es 
preponderante la presencia de estudiantes de tiempo completo y sus padres cuentan con estudios universitarios, mientras que en las instituciones públicas, aunque la mayoría de los estudiantes son de tiempo completo, una considerable proporción trabaja medio tiempo y tiempo completo en áreas no relacionadas con su carrera, cursaron su educación básica en instituciones públicas y la mayoría de sus padres no tuvieron acceso a la universidad, son "pioneros" (De Garay, 2002). En 2007, la proporción de estudiantes inscritos en universidades privadas era importante, 33.2\% (712,648) (ANUIES, 2007).

Las universidades privadas que consideró De Garay son "instituciones que cuentan con una importante trayectoria, reconocimiento y prestigio académico en cada una de las regiones", es decir, instituciones de élite. Es importante esta diferencia dentro de las instituciones privadas, pues existe una gran proporción que puede ser clasificada como instituciones de absorción de las demandas estudiantiles (IAD). Según Gandarilla (2007: 62), estas instituciones se caracterizan por dedicarse exclusivamente a la docencia de licenciaturas altamente demandadas, contar con infraestructura mínima, profesores sobre cargados de actividades docentes en una o más instituciones y/o con actividad profesional ajena a la investigación, y colegiaturas accesibles a la mayoría de los aspirantes rechazados de las universidades públicas. Así que entre los estudiantes de las universidades privadas también habría dos mercados de oferta segmentados, con valor desigual en el mercado y con restricciones de acceso a cada uno.

Las opciones más baratas del sistema privado, debido al bajo costo de las matrículas y a los pocos recursos con los que funcionan, "se caracterizan por una educación de menor valor en calidad y en la rentabilidad del título, que muchas universidades del sector público". (García Guadilla, 2001: 82). Considerando las definiciones de Gandarilla (2007), García (2001) y Mendoza (1998), hice un cálculo propio de la cantidad de estudiantes inscritos en IAD en el país, según la información de la SEP (1999) y ANUIES (2003), y con la información de las páginas web de 48 instituciones privadas con más de 5,000 estudiantes obteniendo los siguientes resultados: 
Cuadro 1 - Matrícula y proporciones en cada tipo de institución privada

\begin{tabular}{|c|c|c|c|c|c|c|}
\hline \multirow[b]{2}{*}{ Universidades de elite y consolidadas } & \multicolumn{2}{|l|}{1995} & \multicolumn{2}{|c|}{1999} & \multicolumn{2}{|l|}{2003} \\
\hline & 191437 & 69.8 & 268096 & 57.2 & 244632 & 39.4 \\
\hline Instituciones de absorción de la demanda & 82749 & 30.2 & 201028 & 42.8 & 375901 & 60.6 \\
\hline Total en instituciones privad as & 274186 & 100.0 & 469124 & 100.0 & 620533 & 100.0 \\
\hline
\end{tabular}

Fuente: Kent y Ramírez, 1998 y cálculo propio con base en SEP, 1999 y

ANUIES, 2003. No existía información correspondiente a 2007.

Respecto al total de la matrícula de licenciatura, la matrícula calculada en IAD en 2003 equivalía al 20.2\%. De manera que una quinta parte de los estudiantes de licenciatura compuesta por jóvenes de escasos recursos han tenido como única opción este tipo de instituciones. Otra información que sería importante conocer de este tipo de instituciones sería la de género. Al menos en el caso de una de las dos IAD que analicé, $75 \%$ eran mujeres.

\section{Cultura regional y creación de IES}

El aspecto central de esta comunicación tiene que ver con la participación de sectores diferenciados de la población en la creación y desarrollo de IES locales y regionales y la participación o el acceso de ciertos sectores a ellas como autoridades, estudiantes y profesores. Me interesa destacar que los grupos culturales y de clase que participan como promotores y como clientes o trabajadores de estas instituciones están ubicados en posiciones diferentes dentro de un campo social en el que las ideologías parcialmente compartidas y legitimadas forman parte importante de la cultura regional. La posición que ocupan se refiere a las redes de relaciones en las que se ubican y las capacidades materiales, sociales y simbólicas con las que cuentan gracias a dichas redes (Lomnitz, 1995). Estas capacidades desiguales de los grupos culturales y de clase en una región juegan un papel importante en la concreción de proyectos educativos ideológicamente orientados.

De entrada, podría considerarse que la participación de diversos actores en la expansión de IES privadas es positiva y favorece la participación política divergente. Sin embargo, una de las tareas centrales de la investigación antropológica es la de proporcionar formas de análisis crítico 
sobre la realidad social. La perspectiva de análisis cultural propuesta puede presentar puntos de vista críticos en dos sentidos. Por una parte, permite mostrar las condiciones político-económicas e ideológicas en que se basa esa dinámica de creación de instituciones educativas (relaciones históricas de desigualdad y competencia). Por otra, muestra algunas probables consecuencias ideológicas, políticas y económicas de dicha dinámica (participación y difusión de ideas del modernismo reaccionario, autoritarismo radical).

Respecto al primer punto, los estudios de caso analizados muestran que a pesar de tratarse de sociedades complejas, existen redes sociales regionales con bases económicas y políticas persistentes. Una forma importante de la concreción y perpetuación de dichas redes se da a través de la fundación y desarrollo de instituciones sociales de diverso tipo. En el caso de Zamora un conjunto de relaciones sociales clave para comprender las características de la Universidad de Zamora fue aquel que se derivó de procesos organizativos regionales de la diócesis de la Iglesia católica con sede en Zamora y de las bases populares y redes familiares de la élite católica regional desde finales del siglo XIX que enfrentó de manera compleja las reformas estatales que concretaron la separación de la Iglesia y el Estado y la secularización de la economía y la política regionales. Cabe destacar que en la región el reparto agrario se concretó de manera tardía en comparación con otras regiones, prolongándose en algunos casos hasta 1937 (Ginzberg, 1999). También es pertinente mencionar que dentro de la política corporativista del nuevo estado post-revolucionario, a pesar de los discursos anti-católicos de algunos líderes nacionales, en la región las bases mantuvieron una ideología católica que supieron negociar localmente (Becker, 2006 y 1994). A finales del siglo XX, el papel de las élites regionales ya no era el mismo en la organización regional del trabajo ni su relación con la Iglesia era tan estrecha en cuestiones financieras. Sin embargo, pudo apreciarse el rol de dichas élites en la creación y desarrollo de una IES promovida por actores locales, en la activación de redes personales y redes institucionales, así como en el funcionamiento de representaciones sociales que otorgaban legitimidad a figuras de la Iglesia católica que apoyaban el proyecto y que ayudaron a obtener la confianza de diversos sectores de la población para su realización. Aunque hubo otro proyecto en esos mismos años para abrir una 
sucursal de la Universidad pública estatal en Zamora, que manejaba un discurso de lucha popular, es sugerente que éste no pudiera concretarse. El proceso inicial de creación de una universidad privada "autónoma de Zamora" dio lugar, mediante la coalición y recomposición conflictiva de un pequeño grupo de actores organizados a través de una asociación civil, a dos instituciones privadas: una sucursal de la Universidad del Valle de Atemajac, originaria de Guadalajara e impulsada por el Arzobispado de esa ciudad, y la Universidad de Zamora. Actores que participaron en la fundación de ambas tenían un discurso a favor de la participación ciudadana que apoya al gobierno en la atención de una demanda social, mediante la creación de instituciones educativas privadas. Otro elemento ideológico y organizativo relevante es la abundancia y extensión en la región de colegios católicos particulares de educación básica y media superior desde antes de los años ochenta en que iniciaron las actividades para la creación de estas dos instituciones ${ }^{3}$.

También es pertinente notar, para apreciar la participación diferenciada de grupos culturales regionales en la creación y desarrollo de estas instituciones, que la asociación civil estaba compuesta por personas que pertenecían a la élite económica o a la clase media, mientras que los alumnos y profesores más bien provienen de estratos medios y bajos, y definitivamente habían tenido procesos de socialización diferentes a los de los hijos jóvenes de los actores pertenecientes al Patronato que creó la UNIVA y la Universidad de Zamora. A pesar de estas diferencias, es interesante notar que la Universidad funciona como un marco de interacción de estos grupos culturales y de clase distintos en los que negocian y comparten parcialmente ideologías atravesadas por elementos de la religiosidad católica. Con respecto a los estudiantes provenientes de clases bajas rurales de pueblos cercanos a la ciudad de Zamora es importante destacar que la amplitud y efectividad de la catequización y el funcionamiento de algunos servicios de atención social de la diócesis de Zamora hizo su aporte a las condiciones que han favorecido el mantenimiento de esta institución con una identidad cristiana que es apoyado ahora por algunos egresados que se incorporaron al trabajo administrativo y al trabajo docente en años recientes.

En el caso de la institución educativa fundada en la ciudad de Zacapu, a $60 \mathrm{~km}$. de Zamora, para comprender sus características resultó pertinente documentar la ausencia de élites regionales con lazos importantes y de larga 
data con alguna institución local relevante para la organización ideológica (Purnell, 1999: 123). Los procesos de constitución del Estado postrevolucionario y el gran apoyo que recibió entre amplios sectores de la población gracias a un proceso de pauperización y despojo de tierras por parte de una empresa agrícola moderna a principios del siglo XX (hacienda), resultaron trascendentales en las condiciones ideológicas y organizativas que permitieron la creación de una IES con un discurso de justicia social y de lucha a favor del "Pueblo".

En este caso, quienes inicialmente apoyaron el proyecto educativo fueron algunos ejidatarios, identidad campesina que surgió con la reforma agraria en los años veinte, y algunos obreros de la planta industrial fundada en los años cuarenta. Durante un tiempo ocuparon la instalaciones de una Casa ejidal, lugar donde sesiona la organización oficial que lleva los asuntos de la Comunidad agraria de una localidad rural del municipio de Zacapu. Posteriormente ocuparon un terreno donado por el municipio y comenzaron la construcción de sus instalaciones con materiales elementales como vigas de madera y láminas. Uno de los recursos gubernamentales iniciales fue dado por Cuauhtemoc Cárdenas, quien fue gobernador del estado en los años 1980-86 y fue visto por los promotores de la IES de Zacapu como un impulsor de proyectos campesinos y populares. A lo largo de los años, han logrado conseguir apoyos gubernamentales con una participación del gobierno local para la gestión de dichos recursos, lo cual ha derivado de una legitimidad local del beneficio público de sus servicios a pesar de que legalmente es una institución privada. Parte de esa legitimidad deriva de su discurso de justicia popular pero también de acciones como la adaptación de sus horarios al de un porcentaje importante de jóvenes que trabajan y estudian y el mantenimiento de cuotas bajas dentro del mercado educativo estatal, inclusive por debajo de algunas escuelas de nivel medio superior.

Intento aclarar que la historia cultural, o la historicidad, de las instituciones no consiste en determinar un hecho precedente como una causa simple de un hecho posterior, lo que nos llevaría a una regresión al infinito. Más bien se propone una forma de precisar la posición de los actores en un campo social y las acciones intencionales para generar coherencia cultural en un sentido determinado por parte de los actores regionales, y de destacar las condiciones desiguales en el manejo y acceso a recursos de distinto tipo. Así 
se comprenden las condiciones que posibilitan, sin ser una causa determinante, la formación contemporánea de IES con todas sus peculiaridades. No resaltar esta intencionalidad y asimetrías sería favorecer mecanismos ideológicos que tratan precisamente de ocultar tales relaciones. Sin ello, inclusive el éxito de una institución podría ser tomado por sus promotores como una "demostración" de que su postura ideológica es la correcta.

Aunque es cierto que las transformaciones del campo social regional pueden derivar de procesos coyunturales externos al campo, tales como la implantación de una zona industrial en un pueblo rural como es el caso del municipio de Zacapu, también, de manera dialéctica, las ideologías interiorizadas previamente por los actores orientan sus intereses y la intencionalidad de sus acciones generan consecuencias en las condiciones económico-políticas a futuro, restringen unas posibilidades de cambio y favorecen otras (al impedir la implantación de una empresa transnacional, por ejemplo). Al analizar las dinámicas regionales se ve que los grupos culturales tienen relaciones jerárquicas que los ubican en ventaja o desventaja frente a otros en el control de recursos y en la legitimidad de sus acciones dentro de la organización social regional.

Este aspecto de la desigualdad de condiciones simbólicas, económicas y políticas es central en el análisis del desarrollo institucional, sobre todo cuando se trata de discutir transformaciones en el acceso a la participación política de distintos actores. En la actualidad se ha desarrollado un discurso en torno a la tolerancia a la diversidad que ha sido retomado por todo tipo de actores en las regiones de estudio como un discurso políticamente correcto aunque de manera superficial, pues se resaltan las diferencias identitarias de los participantes y se pasan a segundo plano las desigualdades entre los mismos, utilizando en ocasiones estrategias de naturalización de dichas diferencias.

Por lo tanto, las consecuencias del desarrollo de instituciones específicas, derivado de esta dinámica cultural con relaciones de desigualdad, nos lleva la discusión de la participación de grupos culturales con proyectos de sociedad divergentes en una misma sociedad nacional. En uno de los casos de mi investigación, la institución promueve que no se cuestionen ciertos principios de orden social y se traten como si fuesen "naturales" y 
recurre para ello a una autoridad incuestionable. A mi parecer, esta situación es fundamental para establecer criterios que permitan esclarecer las consecuencias de la participación de ciertos grupos culturales respecto a las posibilidades de fortalecer subjetividades cívicas críticas, que difundan un pensamiento fundamentado en el conocimiento de la relatividad histórica de las ideas y en el cuestionamiento a la naturalización de relaciones desiguales que en realidad son construcciones sociales e históricas.

Al reflexionar en torno al rol de la secundaria pública para promover relaciones de equidad entre los géneros, que vaya más allá de un discurso de igualdad formal, Levinson (1999: 29) propone una democratización curricular de la educación secundaria en la que se analice lo que la masculinidad ha significado y representado para los grupos oprimidos por ella: un currículo inclusivo para "desarrollar las capacidades de empatía y habilidades para la comprensión de las perspectivas de los demás" (29). De manera que se reconoce la capacidad de las instituciones de educación formal de todo tipo para orientar ideológicamente en algún grado a los sujetos que está formando, pero esto puede ocurrir en muy diversos sentidos.

Quiero ilustrar dos posturas contrastantes con una sola representación ideológica de cada una de las instituciones analizadas. En el caso del Centro Universitario del Valle de Zacapu (CUVZ) lo haré con base en la discusión y reflexión que hacen en torno a la categoría de "El Pueblo" o "el pobrerío". Mientras que en el caso de la Universidad de Zamora lo haré con la cuestión de las relaciones entre los géneros.

El proyecto de la IES de Zacapu tenía originalmente como misión atender necesidades de educación superior de la gente más necesitada de la región, a la que se referían con el término de "El Pueblo o "el pobrerío" y se objetivaba en el lema de la institución: "La Universidad al Pueblo", tomada del título de uno de los murales de David Sequeiros ubicado en Ciudad Universitaria de la UNAM, institución donde hicieron sus estudios las dos fundadoras del CUVZ. Lo que me interesa destacar es el discurso reflexivo que en el año 2004, a veinte años de la fundación de la escuela, realizaban en torno al logro de dicha misión. Las fundadoras reconocían que la mayoría de los estudiantes que estaban en la actualidad asistiendo a la institución no provenían de los estratos más humildes del municipio de Zacapu y hacían una reflexión sobre todos los "filtros" socio-económicos que debían traspasar los 
estudiantes con menores recursos económicos antes de concluir la educación media superior. Ante esa situación volvieron a intentar, como lo habían hecho veinte años antes, que la institución que habían creado fuese convertida en una sucursal de alguna institución pública del estado con la finalidad de que se anulara el costo de las colegiaturas y aumentaran un poco las posibilidades de continuar sus estudios para dicho sector.

Más que discutir la viabilidad o lo adecuado de sus estrategias, en este momento me interesa resaltar que las fundadoras realizaban un constante análisis de las cambiantes situaciones de su realidad local y la adaptación o desajuste entre esa realidad y el discurso y los principios de orden de la institución. Aunque en el caso del tratamiento que daban a las diferencias étnicas se apreciaba una naturalización de las culturas étnicas, en la institución estaba presente de manera sistemática una revisión hacia sus propias formas de representación del mundo social.

En contraste, entre los promotores de la Universidad de Zamora lo que predominaba era un universo discursivo que privilegiaba la distinción de representaciones de la realidad social "adecuadas", universalmente legítimas. El caso de las relaciones de género no era la excepción. Cotidianamente se emitían y se institucionalizaban discursos, por ejemplo en la misión de la carrera de psicología, en torno a la naturaleza de cada género: masculinoracional y femenino-afectivo, subrayando la misión del hombre para predominar en el espacio público y la maternidad como misión predominante de la mujer. Se fundamentaba esta perspectiva en una postura teológica sobre la realidad humana. Al respecto, Rebel rastrea el origen de un pensamiento contemporáneo que surgió en el siglo XVI y que denomina modernismo reaccionario, que se opone a una visión dialéctica de la realidad y de la misma conciencia humana, y que busca un orden legitimado por una razón a-histórica, trascendental (Rebel, 1998). Las ideas promovidas de manera sistemática por la Universidad de Zamora se encuentran dentro de esta línea de pensamiento.

En otra obra (Ávila, en prensa) presento diversos ejemplos de estos discursos y otros recursos simbólicos con los que se promueve una visión de mundo peculiar dentro de cada una de las instituciones así como la postura de sus promotores frente a las inadecuaciones de sus ideas a las circunstancias que en esos momentos estaban enfrentando. Por el momento 
sólo pretendo destacar los contrastes ideológicos existentes entre dos IES fundadas por actores locales fundamentadas en redes de relaciones sociales regionales que implican a la vez recursos simbólicos materiales y sociales (formas de organización, acceso a la información a través de ellas, etc.). Las consecuencias del funcionamiento efectivo, en términos ideológicos y de prácticas compatibles con tales ideologías, tiene mucho que ver con esos fundamentos regionales que proporcionan condiciones favorables para cada uno y que no pueden ser modificados con estrategias en el plano de las ideas exclusivamente, por ejemplo mediante programas nacionales de "educación para la democracia", pues tales programas son en realidad adaptados y limitados por las culturas regionales en las que se aplica.

\section{Comentarios finales}

A mi parecer, una tarea fundamental de las ciencias antropológicas consiste en proporcionar formas de crítica en el sentido propuesto por Leal (2003), como una forma de precisar o discernir la relatividad histórica de las ideas o de la razón humana, así como precisar las posibilidades naturales (neuronales, genéticas) del pensamiento humano. La tarea de la crítica en este sentido es la de discernir que los conocimientos de que somos capaces

no están solamente determinados por la génesis y estructura naturales de las que partimos y que son un producto de la evolución sino también están determinados por la clase de sociedad a la que pertenecemos, por el nivel tecnológico que alcanzamos, por la estructura del sistema industrial y comercial en que nos desenvolvemos, por el lugar que en todas esas relaciones ocupamos: estos modos de determinación sociales resultan tan importantes como los modos de determinación naturales, y es igualmente urgente estudiarlos e investigarlos (2003: 256).

Una estrategia recurrente de distintos actores en todo momento ha sido precisamente la de ocultar ese carácter histórico de los proyectos de sociedad que promueven. El tipo de análisis desarrollado en la investigación, al insistir en las bases ideológicas, económicas y políticas que se han generado entre diversos grupos culturales en una región a través de procesos históricos previos, nos permite señalar las condiciones de posibilidad de que la política ontológica de cada grupo o coalición de grupos culturales y de clase prevalezca sobre otros. Es decir, aclara las acciones ideológicamente orientadas con las que los actores se esfuerzan por modificar la realidad 
social de un modo acorde con sus intereses y con sus representaciones de la misma. Insistir en la historicidad de las representaciones e ideologías de dichos proyectos, permite contar con una forma de crítica más allá de una simple oposición entre 'izquierda' y 'derecha' o 'progresista' y 'reaccionario', y proporcionar una comprensión del desarrollo histórico de la negociación que se va dando entre distintos grupos en torno al significado de ciudadanía, equidad y proyecto de sociedad nacional.

\section{Notas}

1 Aunque la relación entre la formación del Estado-nacional y el sistema educativo público está presente en el origen de todas las naciones modernas, y por tanto en toda Latinoamérica, uno de los indicios para considerar en la actualidad la importancia ideológica del sistema de educación pública son las cifras acerca de la distribución de matrícula entre escuelas públicas y privadas. Destacan México, Bolivia y Brasil, con más de $80 \%$ de la matrícula de educación básica en zonas urbanas dentro del sistema público (Pereyra, 2008).

2 Apenas en 2005 se creó otra universidad pública estatal que pretende favorecer la desconcentración de estos servicios, la Universidad de la Ciénega.

3 El estudio de Torres permite identificar un proceso de amplia participación de la iglesia católica en la escolarización del occidente del país desde la colonia hasta nuestros días (1998), con declives pero con una continuidad clara (1998).

\section{Referências}

AFONSO, Almerindo Janela \& LUCIO-VILLEGAS, Emílio (2007). Estado-nação, educação e cidadanias em transição, Revista Portuguesa de Educação, vol 20, ํㅜ 1, pp. 77-98.

ANUIES (2007). Anuarios Estadísticos 2004-2007. [Consultado: 10/09/2008, Disponible en: http://www.anuies.mx/servicios/e_educacion/docs].

ANUIES (2003), Estadísticas de educación superior, México, ANUIES.

ANUIES, (2001). Estadísticas de educación superior. México: ANUIES.

ÁVILA, Arturo (en prensa). Educación superior privada en la construcción regional del México Neoliberal. México: IPN.

BECKER, Marjorie (2006). Becoming Campesinos: Politics, Identity, and Agrarian Struggle in Postrevolutionary Michoacán, 1920-1935, Hispanic American Historical Review, vol 86, ํo 2, pp. 392-394. 
BECKER, Marjorie (1994). Torching la purisima and dancing at the altar. In G. Joseph and D. Nugent (eds.), Everyday Forms of State Formation: The Negotiation of Rule in Modern Mexico. Durham: Duke University Press.

BRACHO, Teresa (2002). Desigualdad social y educación en México. Una perspectiva sociológica, Educar, 29.

CORREIA, Luciana; CASTRO, Maria Amélia \& GOUVÊA, Maria Cristina (2007). Movimentos sociais e experiência geracional: a vivência da infância no Movimento dos Trabalhadores sem Terra, Educação em Revista no 46, pp. 133156.

DAVIES, Ian; EVANS, Mark \& REID, Alan (2005). Globalising Citizenship Education? A Critique of 'Global Education' and 'Citizenship Education'. British Journal of Educational Studies, vol 53, № 1, pp. 66-89

DE GARAY, Adrián (2002). Un sistema de educación superior, dos realidades distintas: la universidad pública y la universidad privada. Revista de la Educación Superior, vol XXXI, no 122, abril-junio.

DE GARAY, Adrián (2001). Los Actores Desconocidos: una Aproximación al Conocimiento de los Estudiantes. México: ANUIES.

EGERTON, Muriel (2002). Higher education and civic engagement. The British Journal of Sociology, vol 53, no 4, diciembre, pp. 603-620.

GANDARILLA, José (2007). Mercantilización y privatización de la educación superior. In J. Gandarilla (comp.), Reestructuración de la Universidad y del Conocimiento, México: CEllCH.

GALVÁN, Luz Elena (2004). Arquetipos, mitos y representaciones en libros de historia patria (1934-1939). In C. Castañeda, L.E. Galván \& L. Martínez (coords.), Lecturas y Lectores en la Historia de México. México: CIESAS, COLMICH, Universidad Autónoma del Estado de Morelos.

GARCÍA GUADILLA, Carmen (2001). Equidad y gratuidad en la educación superior venezolana. Algunos elementos para la discusión. Revista de la Educación Superior. México: ANUIES, 117, ene-mar, pp. 79-85.

GEERTZ, Clifford (1973). The Interpretation of Cultures. New Cork: Basic Books.

GINZBERG, Eitan (1999). Lázaro Cárdenas: Gobernador de Michoacán, 1928-1932. Zamora: COLMICH, UMSNH.

GONZÁLEZ, Rosario (2008). Formación ciudadana en la escuela pública. Contribuciones y Retos. Comunicación presentada en la Mesa de trabajo: Educación, ciudadanía y cultura política en México: siglo XX, Zamora: COLMICH, 28 y 29 agosto.

HOLLAND, Dorothy \& LAVE, Jean (1996). History in Person. Enduring Struggles, Contentious Practice, Intimate Identities. (School of American Research Advanced Seminar Series).

JIMÉNEZ, Robert, \& SMITH, Patrick (2008). Mesoamerican Literacies: Indigenous Writing Systems and Contemporary Possibilities. Reading Research Quarterly, vol 43, no 1, pp. 28-46. 
KENT, Rollin \& RAMÍREZ, Rosalba (1998). La educación superior en el umbral del siglo XXI. In P. Latapí (coord.), Un siglo de Educación en México. México: Fondo de Cultura Económica.

LEAL, Fernando (2003). ¿Qué es crítico? Apuntes para la historia de un término. Revista Mexicana de Investigación Educativa, enero-abril, vol 8,nำ1, pp. 245261.

LEVINSON, Bradley (1999). Ideología de género en una escuela secundaria mexicana: hacia una práctica institucional de equidad. Revista Latinoamericana de Estudios Educativos, vol 29, ํㅡㄹ, pp. 9-36.

LOMNITZ, Claudio (1995). Las Salidas del Laberinto. México: Joaquín Mortiz.

MENDOZA, Javier (1998). La educación superior privada en México. In P. Latapí, (coord.), Un siglo de educación en México, México: FCE.

PEREYRA, Ana (2008). La fragmentación de la oferta educativa: la educación pública vs. la educación privada. Boletín SITEAL no 8, Buenos Aires: SITEAL. [Consultado el 12, septiembre, 2008, disponible en http://www.siteal.iipeoei.org].

PURNELL, Jenie (1999). Popular Movements and State Formation in Revolutionary Mexico, Durham: Duke University Press.

REBEL, Hermann (2004). Después del siguiente genocidio: el modernismo reaccionario y el desafío postmoderno a la ética analítica. Relaciones vol XXV, no 98.

RíOS, Herculano (2001). La desconcentración de la Educación Superior en cifras. Revista de la Educación Superior, no 120.

ROCKWELL, ELSIE (2007). Hacer Escuela, Hacer Estado. La Educación Posrevolucionaria Vista Desde Tlaxcala. Zamora: COLMICH, CIESAS, CINVESTAV.

ROCKWELL, Elsie (2001). The uses of orality and literacy in rural Mexico. En D. Olson \& N. Torrance (eds.), The Making of Literate Societies, Oxford: Blackwell.

ROSEBERRY, William (1982). Balinese cockfights and the seduction of anthropology. Social Research, vol 49, no4, pp. 1013-1028.

ROTH, Andrew (2007). La amenaza cultural mexicana en dos tradiciones literarias. El Cotidiano, vol 22, no 143, pp. 86-91.

SEP (2003). El Sistema Educativo de los Estados Unidos Mexicanos. Principales Cifras. Ciclo escolar 2002-2003. México: SEP.

SEP (1999). Estadísticas de Educación Superior, Inicio de Cursos. México: SEP.

TORCHE, Florencia (2007). Movilidad Intergeneracional y Cohesión Social: Análisis Comparado de Chile y México. Proyecto Nacsal Cieplan-IFHC.

TORRES, Valentina (1998). La Educación Privada en México, 1903-1976, México: El Colegio de México, UIA.

WOLF, Eric (1982). Europe and the People without History. Berkeley: University of California Press. 


\section{PROJETOS EDUCATIVOS REGIONAIS E PROJETOS DIVERGENTES DA NAÇÃO}

Resumo

Propomo-nos avaliar a participação de actores regionais na construção de cidadanias nacionais com nuances regionais por meio da criação das instituições da instrução superior do regime particular. Os projectos da nação dos actores regionais têm uma base social regional cuja historicidade é construída com os processos de luta entre grupos regionais de classe e na interacção com os agentes do governo nacional. Tentamos mostrar que os princípios promovidos no interior das instituições sobre a realidade social e política, o significado do compromisso político e justiça estão limitados aos resultados contrastantes em cada região de processos de luta de duração longa, como a concretização dos factos entre grupos regionais culturais e da classe durante a consolidação do estado post-revolucionário.

Palavras-chave

Educação superior; Cultura regional; Estado-nação 
REGIONAL EDUCATIVE PROJECTS AND DIVERGENT PROJECTS OF NATION

\section{Abstract}

We propose to value the participation of regional actors in the construction of national citizenships with regional shades by means of the creation of institutions of superior education of private regime. The projects of nation of the regional actors are founded on a regional social base whose historicity has been constructed through conflicting processes between regional groups of class and in interaction with agents of the national government. We try to show that the principles promoted to the interior of the institutions on the social and political reality, the meaning of the political commitment and justice, are bound to the divergent results in each region of conflicting processes of long duration, like the concretion of pacts between cultural regional groups and of class during the consolidation of the post-revolutionary State.

Key words

Private higher education; Regional culture; Nation-state

Recebido em Setembro/2008 Aceite para publicação em Fevereiro/2010

Toda a correspondência relativa a este artigo deve ser enviada para: Luis Arturo Ávila Meléndez, CIIDIR Michoacón IPN, Justo Sierra 28, Centro, Jiquilpan, Mich. CP. 59510, México. E-mail: avilart48@hotmail.com 